\title{
Calculando el riesgo de insolvencia, de los métodos tradicionales a las redes neuronales artificiales. Una revisión de literatura
}

\section{Calculating the risk of insolvency, from traditional methods to artificial neural networks. A literature review}

Maryann Katherine Ludeña Dávila

Universidad del Azuay, Cuenca, Ecuador

mludenamaf@es.uazuay.edu.ec

https://orcid.org/0000-0001-7408-0400

Luis Bernardo Tonon Ordóñez

Universidad del Azuay, Cuenca, Ecuador

ltonon@uazuay.edu.ec

http://orcid.org/0000-0003-2360-9911

Recepción: 22/06/2021 | Aceptación: 16/08/2021 | Publicación: 10/09/2021

Cómo citar (APA, séptima edición):

Ludeña Dávila, M. K., y Tonon Ordóñez, L.B. (2021). Calculando el riesgo de insolvencia, de los métodos tradicionales a las redes neuronales artificiales. Una revisión de literatura. INNOVA Research Journal, 6(3), 270-287. https://doi.org/10.33890/innova.v6.n3.2021.1790

\section{Resumen}

En la administración financiera de toda organización el cálculo del riesgo de insolvencia se ha convertido en un parámetro importante, buscando anticiparse a la eventualidad de llegar a tener un problema económico y generar insolvencia. El objetivo de este trabajo es determinar si en el cálculo del riesgo de insolvencia, el uso de redes neuronales artificiales genera mejores resultados que las metodologías tradicionales, buscando las principales características dentro de las aplicaciones realizadas por distintos autores a través del tiempo. De esta manera se observan las principales variables que pueden evidenciar que la aplicación de la metodología de redes neuronales facilita el cálculo del riesgo de insolvencia. A través de la revisión bibliográfica, en el período 1992-2021, con el uso del método analítico-sintético se puede evidenciar que el modelo expuesto es considerado como eficiente según sus resultados, con ajustes que, en la mayoría de los casos expuestos, superan el $80 \%$ de eficacia. Los resultados encontrados permitieron concluir que 
Calculando el riesgo de insolvencia, de los métodos tradicionales a las redes neuronales artificiales. Una revisión de literatura

la estructura básica de una red neuronal viene dada por tres capas: una de entrada, una de salida y una oculta. Sin embargo, el número de nodos es el que varía en cada una de las aplicaciones realizadas por los distintos autores, dado que los mismos representan a las variables, en este caso indicadores financieros más relevantes según la aplicación planteada. Finalmente se logró evidenciar cuáles son los indicadores financieros más usados en las distintas aplicaciones de redes neuronales. Todo indica que las redes neuronales generan resultados más efectivos que los métodos tradicionales.

Palabras claves: riesgo de insolvencia; red neuronal; predicción; fallas empresariales; modelos de predicción.

\begin{abstract}
In the financial management of any organization, the calculation of the risk of insolvency has become an important parameter, seeking to anticipate the eventuality of having an economic problem and generating insolvency. The objective of this work is to compare the classical methodologies and the artificial neural networks applied to calculate the risk of insolvency, looking for the main characteristics within the applications carried out by different authors over time. In this way, the main variables that can show that the application of the neural network methodology facilitates the calculation of the risk of insolvency are observed. Through the bibliographic review, between the years 1992-2021, with the use of the analytical-synthetic method, it can be seen that the exposed model is considered efficient according to its results, with adjustments that, in most of the exposed cases, exceed $80 \%$ efficiency. The results found allowed us to conclude that the basic structure of a neural network is given by three layers: an input layer, an output layer and a hidden layer. However, the number of nodes varies in each of the applications carried out by the different authors, since they represent the variables, in this case the most relevant financial indicators according to the proposed application. Finally, it was possible to show which are the most used financial indicators in the different neural network applications.
\end{abstract}

Keywords: insolvency risk; neural network; prediction; business failures; prediction models.

\title{
Introducción
}

Según Brigham y Ehrhardt (2007) en Estados Unidos de América los diferentes factores económicos que causan la insolvencia empresarial están ligados en el 37,1\% de los casos a su ubicación y tipo de industria, por factores financieros tales como apalancamiento financiero el $47,3 \%$ y $14 \%$ por temas de fraude, error de juicio y mala gestión financiera, entro otros.

La predicción de insolvencia para las empresas es una herramienta fundamental para enfrentar las diferentes crisis que se puedan presentar (Orellana et al. 2020), evidenciando a tiempo las distintas aristas que podrían llevar a un problema financiero y futura insolvencia frente a los patrones que se puedan manifestar, la adecuada selección de variables y gestión del riesgo pueden ayudar a tomar las decisiones correctas a tiempo para evitar un declive empresarial. Por lo que, es importante conocer las metodologías que se podría aplicar para predecir a tiempo la insolvencia empresarial. 
A través del tiempo se ha evidenciado, en distintas ramas del conocimiento, el avance tecnológico que la investigación informática ha generado y cómo este ha ido influyendo en la toma de decisiones gerenciales sobre todo en finanzas. En 1981 se realizó la primera aplicación de redes neuronales en el área financiera, así como la predicción de variables para llevar a cabo dicha metodología, incrementándose a partir de allí los estudios con su aplicación, dado que se aprovecha la singularidad de las redes neuronales realizan un aprendizaje, lo cual ha tenido una amplia aplicación en el campo financiero, sobre todo en temas relacionados con la predicción de quiebra o rendimiento (Del Carpio Gallegos, 2005).

Kumar y Ravi (2007) realizaron una revisión de metodologías que se aplicaron en distintos trabajos, tesis y artículos científicos dando como resultado que en una muestra de 25 artículos en 15 de ellos se aplican técnicas inteligentes usando principalmente razones financieras. La presente investigación tiene como objetivo determinar si en el cálculo del riesgo de insolvencia, el uso de redes neuronales artificiales genera mejores resultados que las metodologías tradicionales, mediante una profunda revisión bibliográfica.

\section{Metodología}

Para este trabajo de investigación se utilizó un método analítico-sintético, incurriendo en un profundo estudio del objeto de investigación que permitió el conocimiento de las principales características: cálculo del riesgo de insolvencia y la aplicación de redes neuronales artificiales para su cálculo. Se obtuvieron los trabajos de los principales autores sobre el cálculo de riesgo de insolvencia, los cuales son considerados como los originadores de las teorías.

En un segundo momento, tomando como referencia el período 2016-2021 y por medio de la información contenida en las bases de datos Scopus, JSTOR, Redalyc y Dialnet se procedió a la identificación de artículos científicos utilizando como términos de búsqueda "riesgo de insolvencia" y "redes neuronales". La búsqueda se realizó sin discriminar el idioma en el que esté escrito el documento o el área geográfica de la cual provenga.

Se obtuvieron 21 referencias de trabajos publicados que resultaron insuficientes para una base de una revisión sólida, por lo que, se decidió ampliar el período de búsqueda iniciando ahora en el año 1992, año en el que aparece la primera referencia de una publicación que cumple con los criterios de búsqueda planteados. Lo anterior justifica que en esta revisión se citen trabajos de más de cinco años de antigüedad. Se obtuvo finalmente un total 287 artículos publicados.

Luego se realizó un filtrado de los resultados mediante la lectura del título y del resumen y luego se realizó un segundo filtrado bajo el criterio de bibliografía más citada o de mayor impacto, organizando las referencias en orden de importancia. Se seleccionó, en primera instancia, una muestra de 30 artículos los cuales fueron fichados para luego organizar la información obtenida utilizando el método inductivo - deductivo partiendo de lo general a lo específico, esperando encontrar diferentes trabajos de investigación que evidencien la evolución y relación de las distintas aplicaciones de redes neuronales.

Los textos que no aportaban al cumplimiento del objetivo planteado fueron sustituidos por los siguientes en orden de importancia. También se decidió ampliar el número de referencias a ser 
Calculando el riesgo de insolvencia, de los métodos tradicionales a las redes neuronales artificiales. Una revisión de literatura

utilizadas tomando en cuenta algunas que por su novedad aún no habían sido citadas por otros autores. El texto final contiene un total de 59 referencias.

\section{Revisión de literatura}

\section{Importancia de la gestión del riesgo de insolvencia}

El riesgo de insolvencia tiene como significado una probabilidad de pérdida, situación adversa o negativa financieramente, resultante de una posición crediticia negativa cuando existe un riesgo de incumplimiento de una de las partes o riesgo de mercado (Caporale et al., 2017), lo que permite suponer que este tipo de riesgo evidenciaría la imposibilidad de pago a terceros y en algunos casos incluiría a los accionistas o socios dependiendo la salud financiera de la empresa. Allen y Gale (2004) lo definen como la técnica de tomar riesgos por rentabilidades mayores y la posibilidad de soportar golpes financieros que afecten la liquidez o rentabilidad. Por otra parte, Thakor (2018), propone que el riesgo de insolvencia se basa en una caída de valor en los activos generando así, la caída de valor en las acciones dejando sin capacidad de endeudamiento a la empresa, por lo tanto, no es de interés para inversionista financiar empresas insolventes o sin capacidad de endeudamiento, llegando a considerarse una cartera de riesgo.

Históricamente, las crisis financieras han generado que las empresas presten atención sobre la importancia de medir el riesgo de insolvencia, indicador que se puede cuantificar mediante información contable y su relación con el mercado, teniendo como objetivo el establecer de esta manera una medida sobre la probabilidad de insolvencia (Lepetit y Strobel, 2015). Por otro lado, Alaka et al. (2017) describen que los diferentes modelos para predecir el riesgo de insolvencia replican variables de estudios anteriores haciendo que su eficacia sea baja, identificar los factores claves para predecir la insolvencia es importante, al existir la falta de guías para la selección correcta de los mismos es necesario generar enfoques acordes al riesgo estudiado, identificando factores con niveles de relevancia, entre ellos se han ratificado los siguientes:

Cuantitativos: siendo estos la liquidez, el apalancamiento, la gestión financiera, la rentabilidad y los flujos de caja.

Cualitativos: entre estos están la toma de decisiones, las estrategias y la sostenibilidad.

\section{Importancia de las herramientas tecnológicas para la predicción de riesgo de insolvencia}

Dinca et al. (2017) manifiestan que la mayor parte de investigaciones de riesgo de insolvencia se basan en estudios multivariables y diferenciales, en donde los resultados obtenidos buscan describir la situación de una empresa, no obstante, las metodologías planteadas presentan varias deficiencias negando la aplicación a las empresas que desean previsión ante un riesgo de insolvencia, pues se destinan más a las que cotizan en bolsa y a la banca; incluso si se pretende la aplicación de algún modelo en otras empresas hacen que la misma sea inasequible al requerir el uso de información contable, la cual los clientes externos no podrían obtener, por lo que se reduce el uso del modelo a una minoría de usuarios como lo serían los gerentes, quienes conocen la salud financiera de la empresa. 
La insolvencia financiera en las empresas genera múltiples consecuencias desfavorables para los interesados como: entes reguladores, analistas económicos - financieros, proveedores, bancos e inversionistas. Estas consecuencias pueden generar gran impacto en otras empresas, la sociedad y la economía. Dicha situación genera importancia en sistemas que manejen alertas financieras antes de que lleguen las empresas a una insolvencia irreversible, por lo que, ha sido de interés el generar modelos que permitan predecir la insolvencia o planificar una solvencia eficiente (Rustam y Yaurita, 2018). Adicional a ello, una predicción de insolvencia es importante en el área financiera de toda empresa, por lo tanto, estos resultados deben ser veraces pues una mala predicción generaría pérdidas económicas al evaluar la situación de la empresa, por lo que, es muy apetecida la automatización de dicha predicción (Obermann y Waack, 2015). Existen veloces cambios en la tecnología y una gran cantidad de métodos y procesos para una planificación de prevención y recuperación de fallas empresariales, pese a que son exigentes y complejos son importantes para la competitividad y el éxito de una empresa (Mohammadian, 2012).

\section{Metodologías tradicionales para predecir el riesgo de insolvencia}

Según Hensher et al. (2007), generalmente se mide el riesgo de insolvencia con los modelos más populares como lo son los lineales, los análisis discriminantes y los análisis logit, pese a que se han desarrollado modelos estructurales o de intensidad, los mismos que son derivados de supuestos empíricos y metodológicos. Limitando a los modelos de elección discreta predominan los econométricos dentro de la investigación de fallas empresariales (Altman, 2001). Los indicadores financieros históricamente constituyen la herramienta más utilizada al momento de gestionar el riesgo de insolvencia, en sus inicios el uso de dichos indicadores servía como punto de referencia para conocer el estado de una organización sana frente a una insolvente; en esta etapa sobre sale el aporte de FitzPatrick (1932) quien, propuso el índice de endeudamiento como una de las principales herramientas para predecir el riesgo de insolvencia. Posteriormente, Beaver (1966) propuso el método estadístico de análisis discriminante univariante obteniendo mejores resultados, principalmente con el uso del ratio de flujo de caja sobre las deudas; Altman (1968) desarrolló los modelos z y z-score los cuales a diferencia del anterior constituyen un modelo multivariante los cuales se basan en una serie de indicadores de liquidez, endeudamiento, rentabilidad, rotación de activos y valor de la empresa entre otros, logrando elevados porcentajes de precisión.

En la literatura existe una amplia gama de modelos para predecir el riesgo de insolvencia, entre ellos podemos encontrar los que aparecieron a finales de la década 1960, bajo la técnica estadística: el análisis discriminante, el análisis discriminante múltiple (MDA), el modelo de regresión logit y el análisis probit. Según Giacosa et al. (2016) en un estudio realizado en empresas italianas los modelos más reconocidos por su base estadística y número de citas, Altman (1983) y Taffler (1983) serían los más relevantes de acuerdo con el análisis discriminante, así como Ohlson (1980) en el análisis logit.

\section{Descripción de características en la aplicación de redes neuronales para el cálculo del riesgo de insolvencia}

Dentro de las técnicas de inteligencia artificial tenemos el método de redes neuronales que mantiene estructuras de percepción multicapa generando un autoaprendizaje, el número de capas y nodos dependerá de las variables de entrada que se requiera en el modelo (López et al., 2014), 
Calculando el riesgo de insolvencia, de los métodos tradicionales a las redes neuronales artificiales. Una revisión de literatura

coincidiendo con Kumar y Ravi (2007) que definen el método como unidades informáticas conectadas entre sí, paralelamente y en capas. Adicionalmente, el método pone en funcionamiento algoritmos de entrenamiento hacia atrás permitiendo garantizar una convergencia y tolerancia de entrenamiento (Wilson y Sharda, 1994) siendo uno de los primeros métodos en ser utilizado por Odom y Sharda (1990) con indicadores financieros desarrollados por Altman (1968), logrando una mejor precisión en los resultados (Atiya, 2001). Su estructura se basa en información de entrada, capas e información de salida, la interrelación que presenten las distintas neuronas de la red depende de la dirección se le da a la misma, es decir, a que irán dirigidos los resultados (Conti et al., 2005). Khediri et al. (2015) explican que una red neuronal artificial está compuesta por una estructura que describe conexiones entre los nodos, la salida de respuesta de un nodo puede ser la entrada de otro y cada nodo es el encargado de transformar una entrada en salida utilizando una función especificada por el modelo, la conexión de los nodos esta dispuesta entre diferentes capas pero no en nodos de la misma capa, la capa de entrada es el ingreso de datos y la de salida es el resultado, cualquier capa entre ellas se denomina capa oculta.

Nasir et al. (2000) realizaron una aplicación de una red neuronal artificial tomando como población a empresas británicas, para los datos de entrenamiento tomaron una muestra de 1350 empresas, para la prueba 400 empresas y finalmente para la validación de la red neuronal un total de 750 empresas. La estructura de la red utilizó 58 nodos de entrada representados en flujos de caja, estados de resultados, balance general, ratios financieros y resumen financiero; con un criterio de selección 0,10 y 0,90 la red obtuvo resultados el $73 \%$ de clasificación correcta de empresas insolventes, un $24 \%$ con desconocimiento y clasificó de manera errada un $3 \%$ de empresas insolventes, por otro lado, desde el punto de vista solvente, la red clasificó correctamente el 34\% de empresas saludables y un 63\% con descocimiento. Como dicho estudio demuestra en el año 2000 aún existían falencias en la aplicación de la red neuronal artificial para predecir el riesgo de insolvencia, por lo que podemos decir que la estructura y selección de las variables se torna clave para el desarrollo de los modelos y el rendimiento de red neuronal.

En el año 2003 se realizó un estudio en Corea del Sur aplicando una red neuronal artificial asociativa (AANN) la cual genera un aprendizaje de identidad de entrada, básicamente se ingresan datos de empresas solventes lo cual permite que la predicción sea mejor a la del sistema basado en ingreso de datos de ambos tipos, es decir, empresas solventes e insolventes. La muestra de entrenamiento fue tomada del periodo 1994-1996 (Solventes 1817/Insolventes 46) con empresas solventes únicamente, no se utilizaron los datos de las empresas insolventes; para la prueba se utilizaron datos del periodo 1997-2000 (Solventes 2285/Insolventes 83). Los indicadores financieros utilizados fueron: Capital de trabajo/Activos totales, Utilidades retenidas/Activos totales, Utilidades antes de interés e impuestos/Activos totales, Capital de mercado/Pasivos totales y Ventas/Activos totales; siendo los nodos de entrada en la estructura, con una capa de entrada, con 3 capas ocultas con función no lineal y finalmente una capa de salida con 5 nodos; dicha estructura dio un rendimiento de la AANN del 80,45\% en comparación con una red neuronal artificial clásica que en dicho estudio presentó un 79,26\% de rendimiento (Baek y Cho, 2003).

En el año 2009 se realizó una aplicación de red neuronal en un caso de Turquía con un conjunto de datos conformado por un total de 65 bancos entre ellos 21 que habían quebrado, con un total de 20 ratios financieros calculados en el mismo periodo de la quiebra, dicho estudio dividió la muestra en un conjunto de entrenamiento y otro para su validación en el año de la quiebra con 
una estructura de red neuronal MLP la cual constaba de 5 capas ocultas con 30 nodos para cada una de sus capas, dicha red clasificó correctamente los datos con una eficacia del 95.,\% en su fase de validación, de esta manera, se considera un resultado satisfactorio (Boyacioglu et al., 2009).

Tseng y $\mathrm{Hu}$ (2010) realizaron una comparación de modelos de predicción en la cual utilizaron una estructura de red neural artificial con datos financieros de FT EXTEL Company Research y DATASTREAM con 904 empresas de Reino Unido de las cuales 353 habrían quebrado entre 1985 y 1994. La estructura planteada se componía por una capa de entrada, una capa oculta y una capa de salida, lo que llama la atención es que las variables de entrada suman un total de 3 nodos, pues se decidió utilizar tres importantes ratios financieros debido a una clasificación significativa entre el análisis de probabilidad de insolvencia y la relación financiera. Los ratios fueron: Capital de trabajo/Gasto operativo, Utilidad después de impuestos/Activo total y Efectivo/Pasivo total. En este estudio se obtuvieron, en la primera corrida, un rendimiento del $80.33 \%$ en el conjunto de entrenamiento y de $75 \%$ en el de prueba. Después de 5 corridas se concluye con un resultado en entrenamiento del $78,69 \%$ y el $87,5 \%$ en prueba.

En el año 2015 se realizó un estudio entre bancos islámicos y bancos convencionales del Consejo de Cooperación del Golfo (CCG) usando métodos tradicionales o paramétricos y algunos métodos inteligentes, entre ellos la aplicación de una red neuronal buscando demostrar la hipótesis: Los bancos islámicos son menos riesgosos que los bancos convencionales. Se utilizó una muestra final de 44 bancos convencionales y 18 bancos islámicos, con 14 ratios financieros. La red neuronal artificial tenía que identificar, mediante coeficientes financieros generados bajo la técnica Pearson, cuáles eran bancos islámicos y cuáles bancos convencionales CCG. (Khediri et al., 2015).

Las características puestas en funcionamiento en la estructura de la red neuronal artificial fueron: una capa de entrada de 14 nodos (14 coeficientes financieros), una regla de clasificación (unidad de salida $\geq 0,5$ dicha observación es G1, por otro lado, si la unidad de salida es $\leq 0,5$ la observación corresponde al G2) y finalmente la capa de salida con 2 nodos como resultados, los cuales se definieron como importancia e importancia normalizada de todas las variables, la importancia de la variable independiente mide cuánto el valor predicho por la red varía para los distintos valores que pueda tener la variable independiente. Dicha red neuronal demostró distintos resultados en cada periodo de aplicación, los cuales fueron: Periodo general (2003-2010) con una eficacia de la red del 87,4\%, periodo antes de la crisis (2003-2006) con un 81,9\% de rendimiento, periodo de crisis (2007-2008) con un $81,8 \%$ y finalmente el periodo posterior a la crisis (20092010) con un $79,7 \%$. El estudio reveló que los bancos islámicos mantienen un menor riesgo de insolvencia. (Khediri et al., 2015).

En Latinoamérica Romani et al. (2002) realizaron una aplicación de la metodología de redes neuronales en empresas chilenas para predecir el riesgo de insolvencia tomando una muestra de datos ajustada de 98 empresas, de las cuales 49 eran solventes y las restantes declaradas como quebradas. La red neuronal se hizo con el algoritmo de "Back-Propagation" utilizando un punto de corte 0,5 y dos grupos de datos, el primero de entrenamiento y el segundo de prueba, con un total de 16 variables por empresa, de las cuales se decidió bajo un análisis discriminante el uso de solo 4 variables como las más importantes (Activo circulante/Pasivo circulante, Pasivo total/Activo total, Pasivo a largo plazo/Patrimonio y el logaritmo natural del total de activos). Los resultados que presentó dicha aplicación fueron de un 95,24\% en cuanto a la clasificación de 
Calculando el riesgo de insolvencia, de los métodos tradicionales a las redes neuronales artificiales. Una revisión de literatura

entrenamiento y un 95,86\% en cuanto a la predicción del riesgo de insolvencia, demostrando así un alto nivel de asertividad con un buen desempeño, clasificación y predicción del mismo.

Finalmente, como el objetivo de la presente investigación es determinar si en el cálculo del riesgo de insolvencia, el uso de redes neuronales artificiales genera mejores resultados que las metodologías tradicionales, un hallazgo relevante es que la estructura planteada en las diferentes redes neuronales estudiadas coincide con la implementación de 3 capas, una de entrada con distintos números de nodos ya que esto depende de las variables seleccionadas para la aplicación, una capa oculta en donde se define el logaritmo de selección y una de salida (Solvente-Insolvente). Así lo menciona Ecer (2013), quien describe que una de las mejores estructuras utilizadas para el cálculo del riesgo de insolvencia es una de 3 capas, una capa de entrada, una capa oculta y una de salida que en este caso propone con 2 nodos de salida para el resultado. Esto nos permite mencionar que lo que cambia, de acuerdo con los autores, es el uso de las variables a emplear tanto para el aprendizaje como para la prueba de dichas redes neuronales ya que las mismas generan los resultados. Por lo que, se considera relevante observar las variables (indicadores financieros) que han utilizado distintos autores. Ver tabla 1.

\section{Tabla 1}

Análisis de variables por autor

\begin{tabular}{|c|c|c|c|c|c|c|c|c|}
\hline Variables & $\begin{array}{c}\text { Back } \\
\text { et al } \\
(1994)\end{array}$ & $\begin{array}{c}\text { Leshno } \\
\text { \& Spector } \\
(1996) \\
\end{array}$ & $\begin{array}{c}\text { Charalambous } \\
\text { et al (2000) }\end{array}$ & $\begin{array}{l}\text { Alam } \\
\text { et al } \\
(2000)\end{array}$ & $\begin{array}{r}\text { Abdelwahed } \\
\text { \& Amir (2005) }\end{array}$ & $\begin{array}{c}\text { Du } \\
\text { Jardin } \\
(2010)\end{array}$ & $\underset{(\mathbf{2 0 1 1})}{\text { Kim }}$ & $\begin{array}{c}\text { Khediri } \\
\text { et al } \\
(2015)\end{array}$ \\
\hline Ingreso/Activo total & $X$ & $\mathrm{X}$ & & $\mathrm{X}$ & & $\mathrm{X}$ & & \\
\hline $\begin{array}{l}\text { Pasivo total/Activo } \\
\text { total } \\
\text { Activo }\end{array}$ & $X$ & & $X$ & & & $\mathrm{X}$ & & $\mathrm{X}$ \\
\hline $\begin{array}{l}\text { financiero/Pasivo } \\
\text { corriente }\end{array}$ & $\mathrm{X}$ & & & & & & & \\
\hline $\begin{array}{l}\text { Pasivo } \\
\text { total/Patrimonio }\end{array}$ & & & & & $X$ & $X$ & $X$ & \\
\hline $\begin{array}{l}\text { Depreciaciones/Activo } \\
\text { fijo }\end{array}$ & & & & & $\mathrm{X}$ & & & \\
\hline $\begin{array}{l}\text { Valores } \\
\text { realizables/Activo } \\
\text { total }\end{array}$ & & & & & $X$ & & & \\
\hline $\begin{array}{l}\text { Efectivo } \\
\text { equivalentes al } \\
\text { efectivo/Activo total }\end{array}$ & & & $X$ & & $X$ & $\mathrm{X}$ & & $\mathrm{X}$ \\
\hline $\begin{array}{l}\text { Pasivo } \\
\text { corriente/Activo total }\end{array}$ & & & $\mathrm{X}$ & & & & & \\
\hline $\begin{array}{l}\text { Patrimonio } \\
\text { neto/Pasivo total }\end{array}$ & & & & & $\mathrm{X}$ & & & \\
\hline $\begin{array}{l}\text { Pasivo } \\
\text { corriente/Pasivo total }\end{array}$ & & & & & $X$ & & & \\
\hline $\begin{array}{l}\text { Pasivo a largo } \\
\text { plazo/Pasivo total }\end{array}$ & & & & & $\mathrm{X}$ & & & \\
\hline Log (Balance) & & & & & $X$ & & & \\
\hline EBE/Activo total & & & & & $\mathrm{X}$ & & & \\
\hline
\end{tabular}




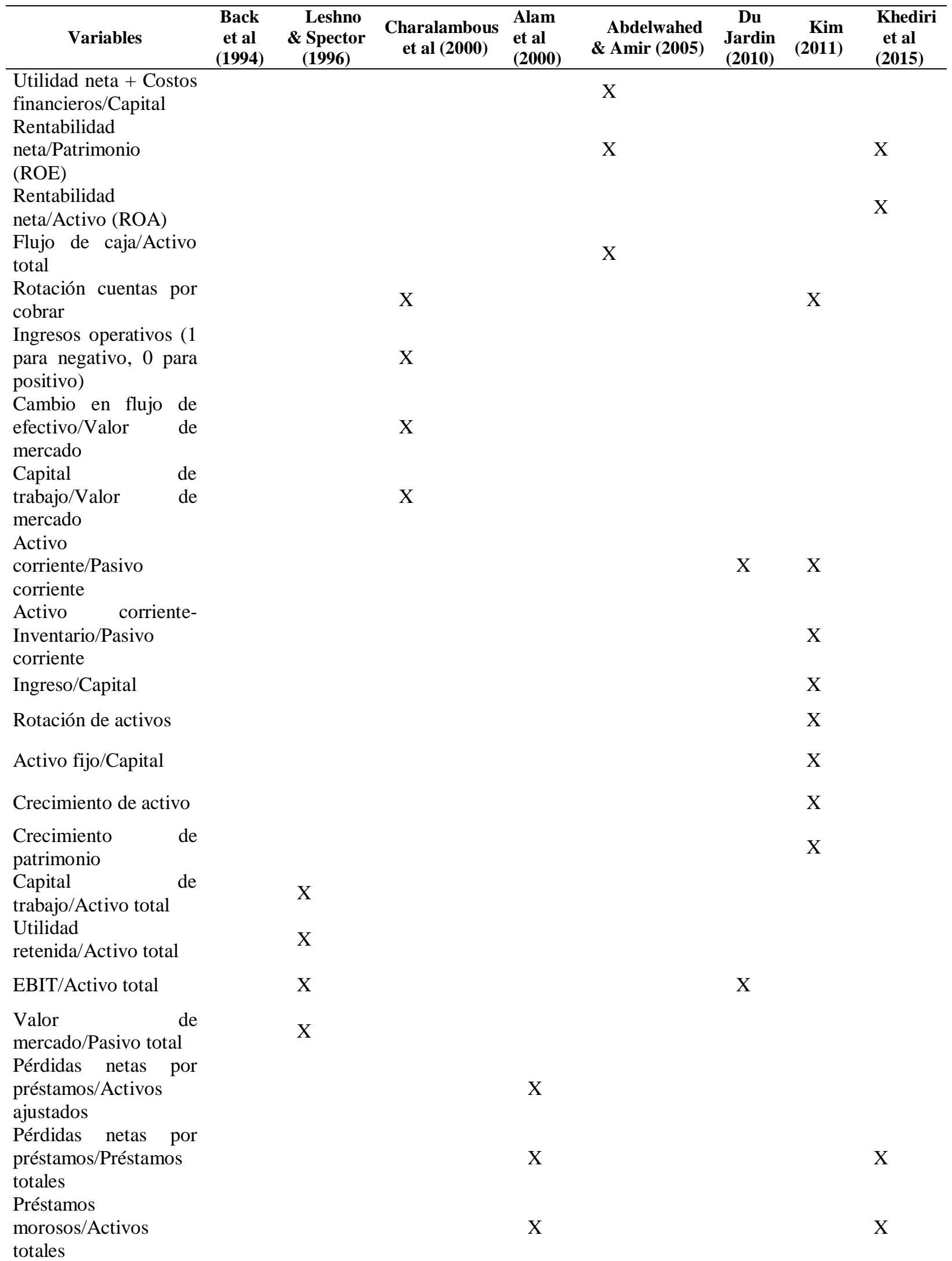


Calculando el riesgo de insolvencia, de los métodos tradicionales a las redes neuronales artificiales. Una revisión de literatura

\begin{tabular}{|c|c|c|c|c|c|c|c|c|}
\hline Variables & $\begin{array}{c}\text { Back } \\
\text { et al } \\
(\mathbf{1 9 9 4}) \\
\end{array}$ & $\begin{array}{c}\text { Leshno } \\
\text { \& Spector } \\
(1996) \\
\end{array}$ & $\begin{array}{l}\text { Charalambous } \\
\text { et al (2000) }\end{array}$ & $\begin{array}{l}\text { Alam } \\
\text { et al } \\
(\mathbf{2 0 0 0})\end{array}$ & $\begin{array}{r}\text { Abdelwahed } \\
\text { \& Amir (2005) }\end{array}$ & $\begin{array}{c}\text { Du } \\
\text { Jardin } \\
(\mathbf{2 0 1 0})\end{array}$ & $\underset{(2011)}{\text { Kim }}$ & $\begin{array}{l}\text { Khediri } \\
\text { et al } \\
(2015)\end{array}$ \\
\hline $\begin{array}{l}\text { (Pérdidas crediticias } \\
\text { netas+ Provisión) / } \\
\text { (Pérdidas } \\
\text { crediticias*ingreso } \\
\text { neto) }\end{array}$ & & & & $X$ & & & & \\
\hline $\begin{array}{lr}\text { Efectivo } & y \\
\text { equivalentes } & \text { al } \\
\text { efectivo/Bancos } & \end{array}$ & & & & & & & & $\mathrm{X}$ \\
\hline $\begin{array}{l}\text { Préstamos } \\
\text { morosos/Préstamos } \\
\text { brutos }\end{array}$ & & & & & & & & $\mathrm{X}$ \\
\hline $\begin{array}{l}\text { Préstamos / Depósitos } \\
\text { totales de clientes }\end{array}$ & & & & & & & & $\mathrm{X}$ \\
\hline $\begin{array}{l}\text { Patrimonio neto / } \\
\text { Activo total }\end{array}$ & & & & & & $\mathrm{X}$ & & $\mathrm{X}$ \\
\hline Bancos/Activo total & & & & & & & & $\mathrm{X}$ \\
\hline Bancos/Capital & & & & & & & & $\mathrm{X}$ \\
\hline $\begin{array}{l}\text { Activo fijo/Activo } \\
\text { total }\end{array}$ & & & & & & & & $\mathrm{X}$ \\
\hline $\begin{array}{l}\text { Partidas fuera de } \\
\text { balance / Activo total }\end{array}$ & & & & & & & & $\mathrm{X}$ \\
\hline EBITDA/Activo total & & & & & & $\mathrm{X}$ & & \\
\hline Cambio patrimonial & & & & & & $\mathrm{X}$ & & \\
\hline $\begin{array}{lr}\text { Efectivo } & y \\
\text { equivalentes } & \text { al } \\
\text { efectivo/Pasivo } & \\
\text { corriente } & \end{array}$ & & & & & & $\mathrm{X}$ & & \\
\hline $\begin{array}{l}\text { Cuentas } \\
\text { cobrar/Ingreso }\end{array}$ & & & & & & $\mathrm{X}$ & & \\
\hline Flujo de caja/Ingreso & & & & & & $\mathrm{X}$ & & \\
\hline EBIT/Ingreso & & & & & & $\mathrm{X}$ & & \\
\hline $\begin{array}{lr}\text { Efectivo } & y \\
\text { equivalentes } & \text { al } \\
\text { efectivo/Ingreso } & \end{array}$ & & & & & & $X$ & & \\
\hline
\end{tabular}

Fuente: Elaboración del autor.

Los autores presentados en la tabla 1, para el desarrollo de cada uno de sus modelos de redes neuronales artificiales aplicadas al cálculo del riesgo de insolvencia han tomado variables definidas previamente en diversas investigaciones relevantes de índole financiero. Como se observa en la revisión bibliográfica del presente artículo, son de gran relevancia las variables usadas para el desarrollo de cada modelo, ya que la estructura de la red neuronal artificial tiene la misma forma básica para este tipo de estudio, pero difieren las variables de entrada utilizadas para el desarrollo del mismo según cada autor. Dentro del análisis se destacan las 4 variables más utilizadas en los distintos modelos, las cuales se mencionan a continuación:

1. Ingreso total/Activo total.

2. Pasivo total/Activo total. 
3. Pasivo total/Patrimonio.

4. Efectivo y equivalentes al efectivo/Activo total.

A través del tiempo se evidencia que a medida que avanza la aplicación de esta herramienta y se muestran sus buenos resultados, también se incrementa el número de variables que se han utilizado en cada modelo, pues hasta el año 2000 los autores citados previamente en la presente, utilizaron un máximo de 7 variables, posterior a ello se han utilizado entre 10 y 15 variables para el efecto. Por lo que, se puede decir que cada vez son más complejas las redes neuronales desarrolladas para el caso y que abarcan un mayor número de aspectos que podrían incidir en un potencial riesgo de insolvencia, todo esto con el afán de incrementar cada vez más la confiabilidad de dicha herramienta.

\section{Discusión}

\section{Ventajas y desventajas de los sistemas basados en redes neuronales artificiales}

Los distintos modelos de aprendizaje automático tienen excelentes resultados en aplicaciones financieras, sobre todo en el cálculo del riesgo de insolvencia por lo que pocos estudios han llegado a alcanzar la efectividad y precisión de estos modelos, los cuales pueden ser aplicados en otros tipos de riesgo ya que generan una precisión sustancial en sus resultados (Barboza et al., 2017).

Dentro de las ventajas que manejan estos sistemas, está la toma de decisiones objetivas que generan los datos y no la mente humana que muchas veces puede tener influencias; al evaluar datos de manera aislada la metodología de redes neuronales no requiere cambios para ser aplicada a distintos tipos de empresas o sectores. (Rustam y Yaurita, 2018). Bešlić et al. (2018) mencionan que estas metodologías están dentro de las más utilizadas ya que proporcionan una señal temprana para poder enfrentar una posible crisis. Lahmiri y Bekiros (2019) demostraron que las redes neuronales son más eficientes al construir agentes artificiales para la predicción de quiebras desde el punto de vista de la informática cognitiva ya que están basadas en datos y son sistemas inteligentes libres de suposiciones lo que las hace altamente capaces de analizar y modelar simultáneamente interacciones de datos ruidosos, no lineales y no estacionarios.

Tay y Shen (2002) después de una revisión de metodologías concluyen que los metodologías modernas son una buena alternativa y prometen buenos resultados con respecto a las convencionales. Por otro lado, Valdes et al. (2014) mencionan que este tipo de simulaciones permiten ir más allá de lo analítico ayudando a tomar decisiones y ver el impacto de las mismas, en un determinado tiempo sin tener que afectar el sistema real o el curso actual de una empresa, algo que las alternativas matemáticas no permiten siendo sofisticadas en su entendimiento y uso. Rubin (1990) identifica una de las diferencias relevantes manteniendo ventaja en el uso de redes neuronales artificiales debido a que utiliza variables predictoras de manera recursiva y jerarquizada. Por otro lado, las metodologás tradicionales usan todas las variables suponiendo una normalidad en las observaciones volviendo más eficiente el uso de la metotología inteligente al considerar valores atípicos en su análisis y parametrización. 
Calculando el riesgo de insolvencia, de los métodos tradicionales a las redes neuronales artificiales. Una revisión de literatura

Alfaro et al. (2008) mencionan que una de las ventajas de la metodologías de redes neuronales artificiales es poder detectar correlaciones y generar buenos resultados ante información ruidosa que suele presentarse en la predicción del riesgo de insolvencia. Dentro de las principales ventajas del uso de redes neuronales Altman et al. (1994) concluyen que, estas logran clasificar con mucha precisión a las empresas con salud financiera o menor riesgo de insolvencia, mejor que un método estadístico. Su uso bajo memoria o aprendizaje ha mostrado una excelente flexibilidad en el modelo permitiendo un rendimiento simple con arquitectura más compleja. Aminian et al. (2006) mencionan que las redes neuronales artificiales brindan un modelo eficaz y eficiente en el análisis de regresiones no lineales y alcanzando en su totalidad el significado de cada variable ingresada en el pronóstico.

Binner et al. (2004) concluyen que los modelos basados en redes neuronales artificiales son más efectivos en los pronósticos a largo plazo, lo que puede servir para distintas proyecciones financieras. El uso de las redes neuronales ha demostrado un alto grado de rendimiento, prometiendo una alerta temprana incluso usando datos limitados en su aplicación y algo destacable, una red neuronal puede ser actualizada sin generar la necesidad de repetir un entrenamiento en la misma (Brockett et al. 1994). Corazza et al (2021) indican que las redes neuronales muestran resultados efectivos y en este contexto son muchos los esfuerzos para el desarrollo de modelos predictivos.

Romani et al. (2002) afirman que de acuerdo con su estudio las redes neuronales artificiales demuestran gran superioridad en rendimiento ante otros modelos como la regresión logit o un análisis discriminante siendo estos modelos estadísticos.

Abedin et al (2018) demuestran, de manera empírica, que al predicir la insolvencia la utilización de redes neuronales probabilísticas genera resultados más robustos que incluso al compararlo con otro método artificial como el basado en una máquina de vectores de soporte. Do Prado et al (2019) para prever la posible insolvencia compararon los métodos de análisis discriminante, regresión logística y redes neuronales, llegando a la conclusión de que el modelo de redes neuronales presentó un mejor resultado. Finalmente, el trabajo de Nadali et al. (2020) al analizar a Pymes en Italia sugiere que el uso de redes neuronales para el cálculo del riesgo insolvencia podría producir resultados mejores en el futuro.

Algunas desventajas, según López et al. (2014) los modelos de redes neuronales mantienen interacciones pequeñas por lo que es necesario eliminar algunas de las variables y tomar las más significativas para el desarrollo, por lo que, consideran que no son mejores que los modelos matemáticos pese a que arrojen resultados dentro de lo aceptable. Rodríguez et al. (2014) desarrollan un modelo de predicción paramétrico de inteligencia artificial para predecir el riesgo de fracaso financiero y determinaron que el modelo predictivo se debilita con el tiempo y requiere una mayor cantidad de variables para obtener un resultado aceptable debido a que las organizaciones analizadas no presentan un perfil claro, derivado de su afán por permanecer en operación, estas asumen medidas que si bien pueden llegar a retrasar el fracaso financiero, generan ciertos desequilibrios y no evitan el fracaso inminente.

Adicionalmente, es necesario mencionar que los resultados dependerán de la calidad de la información financiera, la cual si no es fidedigna puede generar una predicción errónea. Pese a que 
el uso de redes neuronales artificiales se evidencia en estudios sobre la predicción del riesgo de insolvencia su mecanismo, estructura y clasificación no son muy bien comprendidas por lo que se ha tornado difícil aprovechar el potencial de esta metodología (Zhang et al., 1999).

Sin embargo, y a pesar de las dificultades que las redes neuronales presentan se ha evidenciado mediante la revisión bibliográfica que existe una amplia aplicación en el pronóstico de variables financieras (Del Carpio Gallegos, 2005). Altman et al. (1994) señalan los principales problemas o desventajas, dado que la metodología no es dominada puede presentar: demora en el aprendizaje, una deficiente configuración y que el análisis de las ponderaciones llega a ser complejo tornando difícil detectar errores.

Por otro lado, Barrow y Crone (2016) mencionan que la aplicación de redes neuronales al tener una etapa de entrenamiento y otra de validación, requiere el mayor número de observaciones posibles y de datos reales para que la estimación sea real y efectiva; por lo que el tener pocas observaciones puede volver pobre la estimación.

\section{Conclusiones}

Los distintos factores que generan el riesgo de insolvencia, así como los indicadores y metodologías que se usan para su cálculo se encuentran establecidos claramente en la literatura y se conocen como tradicionales, pero a lo largo de esta investigación se pudo evidenciar una rama muy amplia de aplicación en el uso de redes neuronales artificiales para dicho cálculo. Hoy en día en un mundo globalizado y tecnológico es necesario incursionar en nuevas metodologías inteligentes que nos permitan ser menos operativos y más eficientes en el uso del tiempo, ya que dichas técnicas inteligentes facilitan el cálculo del riesgo de insolvencia demostrando un alto grado de efectividad en los resultados que presentan frente a las tradicionales, permitiendo ir más allá de una predicción simple, dando acceso a una toma de decisiones y planificación de estrategias anticipadas para prevenir una insolvencia total de las empresas. Por lo que, según su amplia aplicación para la predicción de riesgo de insolvencia, además de los resultados citados a lo largo de la revisión bibliográfica, se puede afirmar que el uso de redes neuronales para el cálculo del riesgo financiero es más efectivo que las metodologías tradicionales debido a su alto grado de efectividad, además de considerar variables en su totalidad, siempre y cuando las mismas sean correctamente aplicadas pues, de ellas depende el buen resultado que presente la red.

El uso de redes neuronales artificiales en las distintas aplicaciones que existen en la literatura, nos permiten estudiar los factores e indicadores de mayor incidencia al momento de establecer el auto-aprendizaje de la red para generar resultados acordes a la realidad de los diferentes tipos de organizaciones, ya que si bien la estructura de la red para el cálculo del riesgo de insolvencia es generalizada, (constando de 3 capas: entrada, oculta y salida), su número de nodos dependerá del número de variables a utilizarse en el modelo, de hecho se podría estudiar las variables de mayor incidencia para cada sector económico en específico debido a sus diferentes estándares de validación ante el nivel del riesgo de insolvencia que presenta dicho sector económico, para posteriormente someter a la red a las etapas de aprendizaje y validación para así poder garantizar la efectividad de la misma. 
Calculando el riesgo de insolvencia, de los métodos tradicionales a las redes neuronales artificiales. Una revisión de literatura

\section{Referencias bibliográficas}

Abdelwahed, T., \& Amir, E. (2005). New evolutionary bankruptcy forecasting model based on genetic algorithms and neural networks. 17th IEEE International Conference on Tools with Artificial Intelligence (ICTAI'05). https://doi.org/10.1109/ICTAI.2005.92

Abedin, M., Guotai, C., Colombage, S., \& Fahmida-E-Moula. (2018). Credit default prediction using a support vector machine and a probabilistic neural network. Journal of Credit Risk, 14(2), 1-27. https://doi.org/10.21314/JCR.2017.233

Alaka, H., Oyedele, L., Owolabi, H., Oyedele, A., Akinade, O., Bilal, M., \& Ajayi, S. (2017). Critical factors for insolvency prediction: towards a theoretical model for the construction industry. International Journal of Construction Management, 17(1), 25-49. https://doi.org/10.1080/15623599.2016.1166546

Alam, P., Booth, D., Lee, K., \& Thordarson, T. (2000). The use of fuzzy clustering algorithm and self-organizing neural networks for identifying potentially failing banks: an experimental study. Expert Systems with Applications, 18(3), 185-199. https://doi.org/10.1016/S09574174(99)00061-5

Alfaro, E., García, N., Gámez, M., \& Elizondo, D. (2008). Bankruptcy forecasting: An empirical comparison of AdaBoost and neural networks. Decision Support Systems, 45(1), 110-122. https://doi.org/10.1016/j.dss.2007.12.002

Allen, F., \& Gale, D. (2004). Competition and Financial Stability. Journal of Money, Credit, and Banking, 36(3).

Altman, E. (1968). Financial ratios, discriminant analysis and the prediction of corporate bankruptcy. Journal of Finance, 23, 589-609. https://doi.org/10.1111/j.15406261.1968.tb00843.x

Altman, E. (1983). Corporate financial distress: A complete guide to predicting avoiding and dealing with failedcy. New York: John Wiley and Sons.

Altman, E. (2001). Bankruptcy, Credit Risk and High Yield Junk Bonds. New York: Blackwell Publishing.

Altman, E., Marco, G., \& Varetto, F. (1994). Corporate distress diagnosis: Comparisons using linear discriminant analysis and neural networks (the Italian experience). Journal of banking \& finance, 18(3), 505-59. https://doi.org/10.1016/0378-4266(94)90007-8

Aminian, F., Suarez, E., Aminiam, M., \& Walz, D. (2006). Forecasting economic data with neural networks. Computational Economics, 28(1), 71-88. https://doi.org/10.1007/s10614-0069041-7

Atiya, A. (2001). Bankruptcy prediction for credit risk using neural networks: A survey and new results. IEEE Transactions on neural networks, 12(4), 929-935. https://doi.org/10.1109/72.935101

Back, B., Oosterom, G., Sere, K., \& Van Wezel, M. (1994). A comparative study of neural networks in bankruptcy prediction. Proc. Conf. on Artificial Intelligence Res. in Finland, 140-148.

Obtenido

de https://citeseerx.ist.psu.edu/viewdoc/download?doi=10.1.1.137.2473\&rep=rep1\&type= pdf

Baek, J., \& Cho, S. (2003). Bankruptcy prediction for credit risk using an auto-associative neural network in Korean firms. In 2003 IEEE International Conference on Computational 
Intelligence for Financial Engineering, 2003., 25-29. https://doi.org/10.1109/CIFER.2003.1196237

Barboza, F., Kimura, H., \& Altman, E. (2017). Machine learning models and bankruptcy prediction. Expert Systems with Applications, 83, 405-417. https://doi.org/10.1016/j.eswa.2017.04.006

Barrow, D., \& Crone, S. (2016). Cross-validation aggregation for combining autoregressive neural network forecasts. International Journal of Forecasting, 32(4), 1120-1137. https://doi.org/10.1016/j.ijforecast.2015.12.011

Beaver, W. (1966). Financial Ratios As Predictors of Failure. Journal of Accounting Research, 4, 71-111. https://doi.org/10.2307/2490171

Bešlić, D., Jakšić, D., Bešlić, I., \& Andrić, M. (2018). Insolvency prediction model of the company: the case of the Republic of Serbia. Economic research-Ekonomska istraživanja, 31(1), 139-157. https://doi.org/10.1080/1331677X.2017.1421990

Binner, J., Gazely, A., Chen, S., \& Chie, B. (2004). Financial innovation and Divisia money in Taiwan: Comparative evidence from neural network and vector error-correction forecasting models. Contemporary Economic Policy, 22(2), 213-224. https://doi.org/10.1093/cep/byh015

Boyacioglu, M., Kara, Y., \& Baykan, Ö. (2009). Predicting bank financial failures using neural networks, support vector machines and multivariate statistical methods: A comparative analysis in the sample of savings deposit insurance fund (SDIF) transferred banks in Turkey. Expert Systems with Applications, 36(2), 3355-3366. https://doi.org/10.1016/j.eswa.2008.01.003

Brigham, E., \& Ehrhardt, M. (2007). Financial Management: Theory and Practice (14 ed.). SouthWestern Cengage Learning.

Brockett, P., Cooper, W., Golden, L., \& Pitaktong, U. (1994). A neural network method for obtaining an early warning of insurer insolvency. Journal of Risk and Insurance, 402424. https://doi.org/10.2307/253568

Caporale, G., Cerrato, M., \& Zhang, X. (2017). Analysing the determinants of insolvency risk for general insurance firms in the UK. Journal of Banking and Finance, 84, 107-122. https://doi.org/10.1016/j.jbankfin.2017.07.011

Charalambous, C., Charitou, A., \& Kaourou, F. (2000). Comparative analysis of artificial neural network models: Application in bankruptcy prediction. Annals of operations research, 99(1), 403-425. https://doi.org/10.1023/A:1019292321322

Conti, D., Simó, C., \& Rodríguez, A. (2005). Teoría de carteras de inversión para la diversificación del riesgo: enfoque clásico y uso de redes neuronales artificiales (RNA). Ciencia e Ingeniería, 26(1), 35-42. $\quad$ Obtenido de https://www.redalyc.org/pdf/5075/507550773006.pdf

Corazza, M., De March, D., \& di Tollo, G. (2021). Design of adaptive Elman networks for credit risk assessment. Quantitative Finance, 21(2), 323-340. https://doi.org/10.1080/14697688.2020.1778175

Del Carpio Gallegos, J. (2005). Las Redes Neuronales en las Finanzas. Revista de la Facultad de $\begin{array}{llll}\text { Ingeniería } \quad \text { Industrial, } & \text { 8(2), }\end{array}$ http://200.62.146.34/bitstream/handle/123456789/1936/industrial_data04v8n2_2005.pdf ?sequence $=1 \&$ is Allowed $=\mathrm{y}$ 
Calculando el riesgo de insolvencia, de los métodos tradicionales a las redes neuronales artificiales. Una revisión de literatura

Dinca, G., Baba, M., Dinca, M., Dauti, B., \& Deari, F. (2017). Insolvency risk prediction using the logit and logistic models: Some evidences from Romania. Economic Computation and Economic Cybernetics Studies and Research, 51(4), 139-157.

Do Prado, J., De Melo, F., Carvalho, G., \& Ribeiro, A. (2019). Analysis of credit risk faced by public companiesin Brazil: an approach based on discriminant analysis, logistic regression and artificial neural networks. Estudios Gerenciales, 35(153), 347-360

Du Jardin, P. (2010). Predicting bankruptcy using neural networks and other classification methods: The influence of variable selection techniques on model accuracy. Neurocomputing, 73, 2047-2060. https://doi.org/10.1016/j.neucom.2009.11.034

Ecer, F. (2013). Comparing the bank failure prediction performance of neural networks and support vector machines: The Turkish case. Economic research-Ekonomska istraživanja, 26(3), 81-98. https://doi.org/10.1080/1331677X.2013.11517623

FitzPatrick, P. (1932). Average Ratios of Twenty Representative Industrial Failures. The Certified Public Accountant, 13-18.

Giacosa, E., Halili, E., Mazzoleni, A., Teodori, C., \& Veneziani, M. (2016). Re-estimation of company insolvency prediction models: survey on Italian manufacturing companies. $\begin{array}{llll}\text { Corporate Ownership and } \quad \text { Control, 14(1-1), } & \text { 159-174. }\end{array}$ http://doi.org/10.22495/cocv14i1c1p1

Hensher, D., Jones, S., \& Greene, W. (2007). An error component logit analysis of corporate bankruptcy and insolvency risk in Australia. Economic Record, 83(260), 86-103. https://doi.org/10.1111/j.1475-4932.2007.00378.x

Khediri, K., Charfeddine, L., \& Yousseef, S. (2015). Islamic versus conventional banks in the GCC countries: A comparative study using classification techniques. Research in International Business and $\quad$ Finance, 33, https://doi.org/10.1016/j.ribaf.2014.07.002

Kim, S. (2011). Prediction of hotel bankruptcy using support vector machine, artificial neural network, logistic regression, and multivariate discriminant analysis. The Service Industries Journal, 31(3), 441-468. https://doi.org/10.1080/02642060802712848

Kumar, P., \& Ravi, V. (2007). Bankruptcy prediction in banks and firms via statistical and intelligent techniques-A review. European journal of operational research, 180(1), 1-28. https://doi.org/10.1016/j.ejor.2006.08.043

Lahmiri, S., \& Bekiros, S. (2019). Can machine learning approaches predict corporate bankruptcy? Evidence from a qualitative experimental design. Quantitative Finance, 19(9), 15691577. https://doi.org/10.1080/14697688.2019.1588468

Lepetit, L., \& Strobel, F. (2015). Bank Insolvency Risk and Z-Score Measures: A Refinement. Finance Research Letters, 13, 214-224. http://doi.org/10.1016/j.frl.2015.01.001

Leshno, M., \& Spector, Y. (1996). Neural network prediction analysis: The bankruptcy case. Neurocomputing, 10(2), 125-147. https://doi.org/10.1016/0925-2312(94)00060-3

Mohammadian, M. (2012). Artificial intelligence applications for risk analysis, risk prediction and decision making in disaster recovery planning. IFIP Advances in Information and Communication Technology, 382, 155-65. https://doi.org/10.1007/978-3-642-33412$\underline{2 \_16}$

Nadali, L., Corazza, M., Parpinel, F., \& Pizzi, C. (2020). Recurrent ANNs for Failure Predictions on Large Datasets of Italian SMEs. In Neural Approaches to Dynamics of Signal Exchanges (Vol. 151, pp. 145-156). https://doi.org/10.1007/978-981-13-8950-4 
Nasir, M., John, R., Bennett, S., \& Russell, D. (2000). Predicting corporate bankruptcy using modular neural networks. In Proceedings of the IEEE/IAFE/INFORMS 2000 Conference on Computational Intelligence for Financial Engineering (CIFEr), 86-91. https://doi.org/10.1109/CIFER.2000.844606

Obermann, L., \& Waack, S. (2015). Demonstrating non-inferiority of easy interpretable methods for insolvency prediction. Expert Systems with Applications, 42(23), 9117-9128. https://doi.org/10.1016/j.eswa.2015.08.009

Odom, M., \& Sharda, R. (1990). A neural network model for bankruptcy prediction. In 1990 IJCNN International Joint Conference on neural networks., 163-168. https://doi.org/10.1109/IJCNN.1990.137710

Ohlson, J. (1980). Financial Ratios and the Probabilistic Prediction of Bankruptcy. Journal of Accounting Research, 18(1). https://doi.org/10.2307/2490395

Orellana, I., Tonon, L., Reyes, M., Pinos, L., \& Cevallos, E. (2020). Riesgos financieros en el sector manufacturero del Ecuador (1.a ed.). Casa editora Universidad del Azuay.

Rodríguez, M., Piñeiro, C., \& De Llano, P. (2014). Determinación del riesgo de fracaso financiero mediante la utilización de modelos paramétricos, de inteligencia artificial, y de información de auditoría. Estudios de economia, 41(2), 187-217. http://doi.org/10.4067/S0718-52862014000200002

Romani, G., Bravo, A., Aroca, P., Aguirre, N., Vega, P., y Carrazana, J. (2002). Modelos de clasificación y predicción de quiebra de empresas: Una aplicación a empresas chilenas. Forum empresarial, 7(1). https://dialnet.unirioja.es/servlet/articulo?codigo $=6230228$

Rubin, P. (1990). A comparison of linear programming and parametric approaches to the twogroup discriminant problem. Decision Sciences, 21(2), 373-386. https://doi.org/10.1111/j.1540-5915.1990.tb01691.x

Rustam, Z., \& Yaurita, F. (2018). Insolvency Prediction in Insurance Companies Using Support Vector Machines and Fuzzy Kernel C-Means. Journal of Physics: Conference Series, 1028(1), 012118. https://doi.org/10.1088/1742-6596/1028/1/012118

Taffler, R. (1983). The assessment of company solvency and performance using a statistical model. Accounting and Business Research, 13(52), 295-308. https://doi.org/10.1080/00014788.1983.9729767

Tay, F., \& Shen, L. (2002). Economic and financial prediction using rough sets model. European Journal of Operational Research, 141(3), 641-659. https://doi.org/10.1016/S03772217(01)00259-4

Thakor, A. (2018). Post-crisis regulatory reform in banking: Address insolvency risk, not illiquidity! Journal of Financial Stability, 37, 107-111. https://doi.org/10.1016/j.jfs.2018.03.009

Tseng, F., \& Hu, Y. (2010). Comparing four bankruptcy prediction models: Logit, quadratic interval logit, neural and fuzzy neural networks. Expert Systems with Applications, 37(3), 1846-1853. https://doi.org/10.1016/j.eswa.2009.07.081

Valdes, M., Aleaga, A., y Vidal, G. (2014). Redes neuronales artificiales en la predicción de insolvencia. Un cambio de paradigma ante recetas tradicionales de prácticas empresariales. Enfoque UTE, 5(2), 38-58. https://doi.org/10.29019/enfoqueute.v5n2.39

Wilson, R., \& Sharda, R. (1994). Bankruptcy prediction using neural networks. Decision support systems, 11(5), 545-557. https://doi.org/10.1016/0167-9236(94)90024-8 
Calculando el riesgo de insolvencia, de los métodos tradicionales a las redes neuronales artificiales. Una revisión de literatura

Zhang, G., Hu, M., Patuwo, B., \& Indro, D. (1999). Artificial neural networks in bankruptcy prediction: General framework and cross-validation analysis. European journal of operational research, 116(1), 16-32. https://doi.org/10.1016/S0377-2217(98)00051-4 